REVIEW ARTICLE

\title{
Functional significance of sleep
}

\author{
H N Mallick \\ Dr Baldev Singh Sleep Laboratory, Department of Physiology \\ All India Institute of Medical Sciences, New Delhi, 110029
}

Indian J Sleep Med 2010; 5.3, 75-80

$\mathrm{W}$ spend about one third of our life in sleep. During last 30 years there has been tremendous increase in the knowledge of the physiology sleep and sleep disorders. We have come to a conclusion that sleep is an essential physiological need state without knowing its biological significance. Sleep probably has multiple functions for both the brain and the body. It is a function that cannot be substituted by quiet rest. If sleep does not serve an absolute vital function, then it is the biggest mistake the evolutionary forces have ever made ${ }^{(1)}$. This review attempts to highlight most of the proposed functions of sleep based on the results of animal and human studies.

\section{Functions of sleep: Clues from sleep deprivation studies in animals and humans}

Over the years we have looked into the functions of sleep indirectly from outcome of sleep deprivation studies. Extreme sleep deprivation in case of rats has produced severe deleterious effects. Total sleep deprivation in rats is fatal after two to three weeks ${ }^{(2)}$. Within days, animals become hyperphagic but lose weight, a state associated with increased heart rate, energy expenditure and body temperature. Animals become debilitated, emaciated, and develop ulcers on the tail and paws. These signs indicate depression of immune function. Total rapid eye movement (REM) sleep deprivation produces a similar syndrome with a longer time course. As only humans are able to accept sleep deprivation voluntarily, it is difficult to dissociate the

\author{
Address for correspondence \\ Dr H N Mallick, MD, Ph.D \\ Dr Baldev Singh Sleep Laboratory \\ Department of Physiology \\ All India Instt. of Medical Sciences \\ New Delhi, 110029
}

effects of stress from sleep deprivation in rats. Many of these signs of sleep loss are observed after hypothalamic damage and concomitant abnormal functioning of the endocrine and immune systems.

The importance of sleep is also evident from the effects of sleep deprivation in humans. Total sleep deprivation is possible only in experimental animals. In humans, sleep deprivation is most often chronic and partial, and is now increasingly recognized as having deleterious effects on human health. In today's $24 \times 7$ society we are sleep restricted. We are not able to fulfil our daily requirement of sleep resulting in a chronic-partial type of sleep deprivation. Sleep deprivation has two consequences which occur without failure. One is invasion of sleep into wakefulness. When individuals are encouraged or enforced to stay awake, sleep pressure builds up and sleep cannot be avoided irrespective of stimulation. Sleep is unavoidable even at the advent of death as many disasters have happened in the past because of sleeplessness. The second important outcome of sleep deprivation is impairment of cognitive performance. When the amount of sleep is chronically less than the required amount $(7-8 \mathrm{~h}$ per night), there are neurobehavioural consequences on sleep physiology and waking functions ${ }^{(3)}$. The neurobehavioural deficits include lapses in attention; slowed working memory, reduced cognitive output and depressed mood. A causal role for reduced sleep duration in adverse health outcomes, remains unclear; but laboratory studies of healthy adults subjected to sleep restriction have revealed adverse effects on endocrine functions, metabolic and inflammatory responses. These results suggest that sleep restriction produces physiological consequences that may be harmful.

When sleep is restricted to $4-6 \mathrm{~h}$ per night, motor driving performance decreases and subjective report of sleepiness increases ${ }^{(4)}$. Behavioural lapses during performance are due to appearance of microsleeps which ranges from $0.5 \mathrm{~s}$ to $10 \mathrm{~s}$ in duration. As sleep loss continues

Indian Journal of Sleep Medicine (IJSM), Vol. 5, No. 3, 2010 
these microsleeps can grow into full blown sleep. This has been viewed as 'wake state instability'. During such states there are moment to moment shifts between wake and sleep mediating neural mechanisms resulting in a confusion and hallucination. Although motivated humans can overcome sleepiness for a short period, they cannot perform at a high level for sustained periods.

In addition to cognitive impairment, sleep loss is commonly associated with wide range of detrimental effects. Cardiovascular disease and hypertension are associated with sleep loss. Short sleep duration of $5 \mathrm{~h}$ or less is associated with greater mortality from coronary artery disease in comparison to $8 \mathrm{~h}$ of sleep ${ }^{(5)}$. Five nights of sleep restriction for four hours each night has resulted in production of pro inflammatory cytokines including IL-1b, IL-6 and IL-17. The values remain elevated during two days of recovery sleep, accompanied by increased heart rate and serum C - reactive protein ${ }^{(6)}$. Short periods of sleep loss at the time of vaccination reduce the effectiveness of immunization. Sleep loss is associated with increased obesity which is linked to reduced leptin levels and increased levels of ghrelin $^{(7)}$. Sleep loss is also associated with diabetes and impaired glucose tolerance.

Results of sleep deprivation studies from animals and humans do not tell directly anything about the functions of sleep. Collectively, these results demonstrate wideranging consequences of sleep loss on physical and cognitive performances and harmful effects on health.

A neural function: It has been advocated that, sleep is of the brain, by the brain and for the brain ${ }^{(8)}$. Although, the most immediate, unavoidable effect of sleep deprivation is cognitive impairment, the entire body benefits from sleep. The brain suffers most from sleep deprivation. It is less clear that the rest of the body suffers as rapidly and significantly from lack of sleep. Although we talk about a muscle that is active or at rest, muscle rest can be achieved during quite wakefulness, and does not seem to require sleep. However, skeletal muscle atonia and penile erection seen during REM sleep indicate nonneural functions of sleep mediated by the brain.

Our sleep cycles pass through stages of non-REM (NREM) and REM sleep. Brain activities during NREM and REM sleep are remarkably different. The EEG of NREM sleep is distinct with slow waves and spindles and the EEG of REM sleep is similar to that of wakeful state. Brain metabolism and temperature are lower during NREM sleep but higher during REM sleep. Therefore the two sleep stages should be viewed differently for their functional significance.

\section{Functions of NREM sleep}

Clues from electrophysiological signs of sleep: The progression of NREM sleep to deeper sleep stages can be better understood from the concurrent changes in EEG waves profiles. The appearance of EEG spindles, signal the beginning of reduced responsiveness to environmental stimuli. These spindles are generated in the thalamic reticular nucleus during NREM sleep. Thalamocortical neurons are inhibited and incoming sensory messages are blocked from reaching the cortex. We have yet to understand the benefits of this environmental disconnection. Sleep spindles, can provide brief trains of depolarizing inputs to targets in the neocortex, which are similar to spike trains, used experimentally to induce long-term synaptic potentiation. It has been shown by Steriade and colleagues that experimental trains of impulses similar to those produced by sleep spindles can produce lasting changes in the responsiveness of cortical neurons ${ }^{(9)}$.

NREM sleep is also known as slow wave sleep (SWS) because of the slowing of the EEG synchronized waves to delta activities. This is one of the unique electrophysiological signatures of the NREM sleep. This spontaneous brain activity during sleep reflects brain functioning that is not influenced by attention and motivational factors that occur during wakefulness. While it is well known that high-amplitude slow waves are abundant in early sleep and decrease across the sleep period, neither their function nor the mechanisms underlying their local and global occurrence are clear. Probably NREM sleep gives the brain the rest. It was suggested recently that sleep slow wave activity may reflect the average strength of cortical synapses, which would increase during wakefulness as a result of plastic processes and decrease during sleep due to a sleepdependent mechanism of synaptic downscaling ${ }^{(10)}$.

Energy conservation: Energy conservation during sleep has been hypothesized to be one of the important functions. Sleep may be adaptive because it conserves energy and suppresses behaviour during portions of the 24 hour day. Brain metabolic rate is correlated with body metabolic rate. Energy consumption by brain is one of the maximum in our body. As NREM sleep is associated with reduced brain energy expenditure, in organism with large brain, sleep may have become more and more important. Elevated metabolism is linked to a number of biochemical changes 
several of which have been linked to sleep control. It has been proposed that sleep was selected to reduce energy demand ${ }^{(11)}$. Carnivores and omnivores may make more use of the energy-conservation theory of sleep. They tend to have more sleep because they generally have safe sleep places and ability to eat meals with high calorific density that may make continuous activity unnecessary ${ }^{(12)}$. Conversely grazing animals may need to spend more time in eating because of the low calorific density of their food. Large herbivores may have evolved reduced sleep amounts because they are more vulnerable to predators than small herbivores. Small herbivores and other mammals may need to maximize sleep amount to conserve energy because their relatively high ratio of surface area to body mass makes it costly to maintain their body temperature ${ }^{(13)}$.

Energy conservation may be particularly important in newborns. Their high ratio of surface area to body mass makes the energy conservation achieved by sleep highly adaptive. Furthermore, animals those are immature at birth benefit from the sleep-induced reduction in exposure to danger. When body size increases and sensory-motor systems mature, young animals derive greater benefits from waking activities, consistent with the developmental decrease in sleep time.

As sleep is meant for saving energy, it should have a molecular link. Adenosine is a good indicator of metabolic states in glia. It can signal increased sleep pressure and initiate sleep. Adenosine release increase in the basal forebrain area after sleep deprivation and modulate sleep via inhibition of cholinergic basal forebrain neurons ${ }^{(14,15)}$.

Sleep time may be related to defence against oxidative stress. A high metabolic rate results in the generation of high levels of reactive oxygen species (ROS) by mitochondria. Smaller animals have higher metabolic rate than larger animals. Sleep deprivation in the rat is accompanied by increased oxidative stress and evidence of membrane disruption in the hippocampus, subcortical brain regions and peripheral tissues ${ }^{(16)}$. The supraoptic nucleus of the hypothalamus shows the highest rates of ROS generation and interestingly this brain region has also the highest rate of protein synthesis. Many of the cells in the body show increased synthesis and decreased breakdown of protein during sleep. Since proteins are the building block needed for cell growth and repair of damage from factors like stress and ultraviolet rays, deep sleep may be truly beauty sleep. The lower metabolic rate and brain temperature during NREM sleep seem to provide an opportunity to deal with the damage done during awake period.

Higher metabolic rates in the brain warrant longer periods of sleep to (i) interrupt ROS-induced damage to brain cells, (ii) facilitate the synthesis and activities of molecules that protect brain cells from oxidative stress, (iii) allow sufficient time for the repair or replacement of essential cellular components in neurons and glia and (iv) deal with other biochemical consequences of waking metabolic activity.

Neurogenesis: New neurons are generated in adult animals in the olfactory bulb, the subventricular zone lining the lateral ventricles, and in the subgranular cell layer of the dentate gyrus of the hippocampus, in a process that produces functional neurons in 3-4 weeks. The neurogenesis is facilitated by exercise and blocked by stress. Short-term (2-3 days) total sleep deprivation, even when other forms of stress are controlled for, also blocks subsequent proliferation of cells in the dentate gyrus ${ }^{(17)}$. Thus, sleep may have a general role in allowing or facilitating neurogenesis.

Hormone secretion: Growth hormone (GH) secretion occurs in pulses throughout the day, but after sleep onset, there are large bursts of GH secretion associated with SWS ${ }^{(18)}$. GH secretion during SWS can amount to twothirds of the total GH secreted in young males.

Sleep is restorative: Search for the function of sleep has been focussed on identifying neuronal resources depleted during wakeful state and replenished during sleep for quite long time. Alternatively harmful substances that accumulate during wake and their removal during sleep have been also looked for.

One hypothesis was that brain glycogen is depleted during waking and restored during sleep, however subsequent work showed that this effect did not occur in all strains of mice suggesting that this could not be a general regulatory mechanisms or function of sleep ${ }^{(19)}$. A number of endogenous substances have been linked to sleep. Cytokines such as interleukin I have been implicated in sleep control. Substances like adenosine, prostaglandin D, a muramyl dipeptide, delta sleep inducing peptide corticostatin, GHRH, oxidized glutathione, uridine, tumour necrosis factor-alpha, oleamide, cholecystokinin, insulin and nitric oxide have significant effects on sleep ${ }^{(20)}$. The relative importance of each of these sleep factors, the pathways by which they interact with each other, their mode of action and roles in sleep physiology needs elucidation.

Indian Journal of Sleep Medicine (IJSM), Vol. 5, No. 3, 2010 
As protein synthesis is decreased on sleep deprivation, changes in brain gene expression that occur between sleep and wakefulness have been looked into. In all species studied (flies, mice, rats, hamsters and sparrows), wakefulness leads to the up-regulation of three categories of transcripts - those involved in: (i) energy metabolism, (ii) cellular stress response, and (iii) synaptic potentiation. By contrast, transcripts expressed during sleep at higher levels are involved in (i) synaptic depression and depotentiation, (ii) synthesis/ maintenance of membranes, and (iii) lipid metabolisms ${ }^{(21)}$.

During learning and interaction with environment there is a net increase in synaptic strength in many brain areas in animals. A net increase synaptic strength at the end of a waking day would result in higher energy consumption, larger synapses that take up precious space, and saturation of the capacity to learn. Cell need to synthesize and deliver cellular constituents ranging from mitochondria to synaptic vesicles to various proteins and lipid which is a major source of cellular stress. Sleep would be necessary to renormalize synapses to a baseline level that is sustainable and ensures cellular homeostasis ${ }^{(21)}$.

\section{The functions of REM sleep}

REM sleep is ubiquitous among mammals. Amazing progress has been made in localizing the mechanisms that generate REM sleep. However, the mystery of REM sleep remains. The vivid dreams human beings experience during REM sleep have made the function of this state most intriguing. We do not know the biologic need that initiates REM sleep. The features of REM sleep include EEG desynchronization, rapid eye movements, ponto geniculo occipital (PGO) waves, skeletal muscle atonia, erection in males and dream state. During REM sleep some neurons fire more intensely than those seen during waking. There are number of neuronal groups which are completely silent during REM sleep. There must be some functional significance of these features. There are many proposed functions of REM sleep.

REM sleep length is strongly correlated with immaturity at birth. Extremely high level of REM sleep at birth and its reduction in adulthood is seen in many terrestrial mammals ${ }^{(22)}$. Neuronal activity is high during REM sleep. This may be a clue that REM sleep state is involved in the brain growth during early developmental period. If REM sleep serves to maintain or establish brain connections during crucial period of development than the purpose of this state later in life needs explanation. It is proposed that
REM sleep stimulates the adult brain to reverse the effects of NREM sleep during subsequent waking period. Throughout the sleep cycle REM sleep follows N3 stage of NREM sleep, which is considered as the deepest stage of sleep is human. It is difficult to awaken from this stage, whereas it is easier to get up from REM sleep. Animals typically awaken spontaneously from REM sleep ${ }^{(23)}$. Animals that are awakened from NREM sleep have poor sensory motor function compared with those awakened from REM sleep $^{(24)}$. Getting up from a more alert stage has substantial advantage over confused state. One of the functions of REM sleep could be to release from NREM sleep to wakefulness.

The duration of REM sleep period in humans progressively increase throughout the sleep period. It is maximum towards the last part of the night, the intensity of REM sleep, the prevalence of erection in males, the vividness of dream reports increase when the night progresses. Even though the body temperature is largely suspended during REM sleep, there is increase in temperature in several brain regions. There is almost complete skeletal muscle atonia as well as periodic occurrence of twitches, and PGO waves. All these vivid features must be serving some functions. It is proposed that penile erection during REM sleep can serve as alternative to erectogenic and psychogenic sexual arousal.

The role of REM sleep in memory consolidation has drawn a lot of attention. Higher cortical functions such as cognition, attention, and memory are rapidly affected by sleep deprivation. Studies have shown that learning and memory are improved by subsequent sleep without repetition of the task, suggesting information processing occurs during sleep. Enhancement of procedural sensory and motor memories is found to be dependent on overnight sleep with equivalent period of wake failing to produce any performance gains. Consolidation of motor skill has been linked to N2 stage of NREM sleep. In contrast both SWS and REM sleep have been associated with memory consolidation for a visual discrimination task ${ }^{(25)}$. Imaging studies have shown depression in activity during NREM sleep and a reactivation during REM sleep in cortical regions involved in a task learned during prior wakefulness $^{(26)}$.

Throughout the sleep cycle, both REM and NREM sleep stages contain numerous distinct electrophysiological events, each of which contribute to unique physiological state. The role of spindles and slow wave activity in memory processing has already been described. The hippocampal theta waves, seen during REM 
sleep in both humans and other animals, greatly facilitate the induction of long-term potentiation (LTP) in the hippocampus, potentiation that is believed to a physiological mediator of memory formation ${ }^{(27)}$. The PGO waves a phasic event during REM sleep have been associated with learning. Fear conditioning in rats have shown to increase the amplitude of elicited P-waves during REM sleep suggesting it as a regulated component of a sleep-dependent mechanisms of learning and plasticity. These PGO waves occur in a phase-locked manner with hippocampal theta wave activity during REM sleep. This has significance because experimental hippocampal stimulation at the peaks of theta waves facilitates LTP. The same stimulation applied at the troughs of the theta waves instead leads to long-term depression of synaptic responses. These findings suggest that natural PGO activity during REM sleep may serve as an endogenous regulator of synaptic plasticity ${ }^{(28)}$.

Administration of protein synthesis inhibitors during REM sleep in rats prevented behavioural improvement following sleep period ${ }^{(29)}$. Several immediate early genes (IEG) are specifically up regulated not only during sleep but also during wake. However learning specific upregulation of zif-268 a plasticity associated IEG during REM sleep has been shown following exposure to an enriched sensorimotor environment ${ }^{(30)}$. In the absence of such exposure there was down regulation during both NREM and REM sleep. These molecular evidence compliments the behavioural, electrophysiological and imaging data on the role of REM sleep memory consolidation

In a recent review the role of REM sleep in memory consolidation has been severely questioned ${ }^{(31)}$. The evidence for REM sleep-dependent consolidation of declarative memories is inconsistent, weak and contradictory. Results from animal studies are confounded by stress effects. Human with brain lesion and pharmacologically induced suppression of REM sleep do not show memory deficit and other human sleep learning studies have produced inconsistent results. However the memory consolidation theory during REM sleep cannot be disowned so cheaply. There are emerging evidence of NREM sleep participating in information processing and synaptic plasticity. We all experience that a disturbed sleep is certainly not conducive for attention and learning. The dream met during last part of night is better remembered where the intensity of REM sleep is highest. Moreover the role of REM sleep in consolidation of procedural memory need not be down played. Sleep-dependent motor learning is associated with a large-scale plastic reorganization of memory throughout several brain regions, allowing skilled motor movements to be executed with precision and pace following sleep ${ }^{(25)}$. These findings hold important implications for understanding the brain basis of perfecting real life skills.

Sleep, as found in mammals, likely has multiple functions. Sleep and sleep deprivation studies have contributed enormously to understand its functional significance. The nature, amount, place, age dependency of sleep, body size and ecological variables indicate the functions of sleep variable across the species. We universally acknowledge that during sleep energy is saved, performance is restored, molecules are compensated and some forms of memory consolidated. But the functions of sleep still remain a mystery.

\section{References}

1. Mignot E. Why we sleep: The temporal organization of Recovery. PLoS Biol 2008; 6:661-69.

2. Rechtschaffen A, Bergmann BM, Everson CA, Kushida CA, Gilliland MA. Sleep deprivation in the rat: I. Conceptual issues. Sleep 1989; 12:1-4.

3. Banks S, Dinges DF. Behavioral and physiological consequences of sleep restriction. J. Clin Sleep Med 2007; 3:519-528.

4. Horne J. Is there a sleep debt< Sleep 2004; 27:10471049.

5. Ikehara S, Iso H, Date $C$, Kikuchi $S$, Watanabe $Y$, Wada $Y$, Inaba Y, Tamakoshi A. Association of sleep duration with mortality from cardiovascular disease and other causes for Japanese man and women : the JACC study. Sleep 2009; 32:295-301

6. Van Leeuwen WMA, Lehto $M$, Karisola $P$, Lindholm $H$, Luukkonen R, Sallinen M, Harma M, Porkka Heiskanen T, Aleniu H. Sleep Restriction increases the risk of developing cardiovascular disease by augmenting proinflammatory responses through II-17 and CRP. PLoS ONE 2009; 4:e4589.

7. Taheri S, Lin L, Austin D, Young T, Mignot E. Short sleep duration is associated with reduced leptin, elevated ghrelin, and increased body mass index. PLoS Med 2004; 1:e62.

8. Hobson A. Sleep is of the brain, by the brain and for the brain. Nature 2005; 437:1254-1256.

9. Steriade $\mathbf{M}$ The intact and Sliced Brain. MIT Press: 2001

10. Tononi G, Cirelli C. Sleep function and synaptic homeostasis. Sleep Med Rev 2006; 10:49-62.

11. Zeplin H, Siegel J, Tobler I. Mammalian sleep. In: Kryger MH Roth T., Dement WC, editors. Principles and practice of sleep medicine. 2005. Philadelphia: Elsevier Saunders.

12. Alison T and Cicchetti D V. Sleep in mammals, ecological and constitutuional correlates. Science 1975; 194:732734 .

Indian Journal of Sleep Medicine (IJSM), Vol. 5, No. 3, 2010 
13. Siegel $\mathbf{J} \mathbf{M}$. Clues to the functions of mammalian sleep. Nature 2005; 437:1264-1271.

14. Porkka-Heiskanen $T$, Strecker RE, Thakkar M, Bjorkum AA, Greene RW, McCarley RW. Adenosine: a mediator of the sleep-inducing effects of prolonged wakefulness. Science 1997; 276:1265-1268.

15. Strecker RE, Morairty $S$, Thakkar MM, et al. Adenosinergic modulation of basal forebrain and preoptic/anterior hypothalamic neuronal activity in the control of behavioural state. Behav Brain Res 2000; 115:183-204.

16. Ramanathan L., Gulyani S., Nienhuis R. \& Siegel JM. Sleep deprivation decreases superoxide dismutase activity in rate hippocampus and brainstem. Neuro Report. 2002; 13:1387-1390.

17. Meerio $P$, Mistlberger RE, Jacobs $B L$, Heller $H C$ and McGinty $D$. New neurons in the adult brain: the role of sleep and consequences of sleep loss. Sleep Med Rev. 2009: 13:187194.

18. Van Cauter E, Kerkhofs $M$, Caufriez A, Van Onderbergen A, Thorner MO, Copinschi G.A quantitative estimation of growth hormone secretion in normal man; reproducibility and relation to sleep and time of day'. J Clin Endocrinol Metab. 1992; 74:1441-1450.

19. Benington $\mathbf{J H}$, Heller HC. Restoration of brain energy metabolism as the function of sleep. Prog Neurobiol 1995; 45:347-360.

20. Opp MR, Krueger JM. Interleukin-1 is involved in responses to sleep deprivation in the rabbit. Brain Res. 1994 639:5765.

21. Tononi $\mathbf{G}$ and Cirelli $\mathbf{C}$. Staying awake puts pressure on brain arousal system. J. Clin. Invest. 2007; 117:36483650 .
22. Tobler I. Phylogeny of sleep regulation. In: Kryger MH, Roth T. Dement WC. Principles and practice of sleep medicine. Philadelphia: Elsevier Saunders 2005:77-90.

23. Horner R.L, Sanford L.D., pack $A$ i and Morrison A.R. Activation of a distinct arousal state immediately after spontaneous awakening from sleep. Brain Res 1997; 778:127-134.

24. Snider F. Towards an evolutionary theory of dreaming. Am J Psychiatry 1986;123:121-136

25. Stickgold $\mathbf{R}$ and Walker M P. Sleep-dependent memory consolidation. Sleep Med 2007; 8:331-343.

26. Maquet $\mathbf{P}$. The role of sleep in learning and memory. Science 2001; 294:1048-52.

27. Cantero JL, Atienza M, Stickgold R, Kahana MJ, Madsen $J R$, Kocsis B. Sleep - dependent theta oscillation in the human hippocampus and neocortex. J Neurosci 2003 23:1089710903.

28. Karashima A, Nakamura $K$, Sato N, Nakao $M$, Katayama $N$, Yamamoto $M$. Phase locking of spontaneous and elicited ponto-geniculo-occipital waves is associated with acceleration of hippocampal theta waves during rapid eye movement sleep in cats. Brain Res 2002; 958:347-358.

29. Smith C, Tenn C, Annett R, Some biochemical and behavioural aspects of the paradoxical sleep window. Can J Psychol 1991; 45:115-124.

30. Reibeiro S, Goyal S, Mello C, Pavlides C. Brain gene expression during REM sleep depends on prior waking experience. Learn Mem 1999, 6:500-508.

31. Siegel J $\mathbf{M}$. The REM-sleep memory consolidation hypothesis. Science 2001;294:1058-1063. 